\title{
RELATIONS BETWEEN PHOTOSPHERIC STRUCTURE AND X-RAY EMISSION
}

\author{
M. KÜRSTER \\ Max-Planck-Institut für extraterrestrische Physik \\ D-85740 Garching, Germany
}

\begin{abstract}
The relation between photospheric and coronal active regions in late-type stars is studied from two different points of departure. First, I report on 5 years of ROSAT X-ray monitoring of the active young $\mathrm{K}$-star AB Dor. I compare the $\mathrm{X}$-ray data with 16 years of $\mathrm{V}$-band brightness monitoring showing a 10-year decline between 1978 and 1989 and a subsequent rise phase. Quite differently, the X-ray flux of AB Dor (while exhibiting strong variability on time scales of minutes to weeks) reveals no pronounced long-term trend over the 5 years of the program. This supports the concept of a saturated corona. Second, I present rotationally modulated ROSAT $\mathrm{X}$-ray light curves of three active stars (AB Dor, CF Tuc, YY Men) and compare them with contemporaneous Doppler images. I demonstrate that it is possible to explain the $\mathrm{X}$-ray light curves by coronal emission regions that are spatially related with photospheric active regions. I discuss the concept of X-ray bright loops connecting the major star spot complexes.
\end{abstract}

\section{Introduction}

Coronal X-ray emission is an excellent tracer of stellar activity. The Sun, a star only moderately active, shows a pronounced variation of its X-ray flux by a factor of $\approx 10$ (soft) or $\approx 100$ (hard) following the solar activity cycle. In contrast, variations of the visual brightness of the Sun are on the order of one permille. If the Sun were at stellar distances, it would be extremely difficult to detect any variability of its disk integrated visual brightness, whereas the cyclic variation of its $\mathrm{X}$-ray luminosity would be evident.

The most active late-type stars such as rapidly rotating pre-main sequence or RS CVn-type objects have $\mathrm{X}$-ray luminosities that exceed the Sun's maximum X-ray output by 3 orders of magnitude. While these ob- 


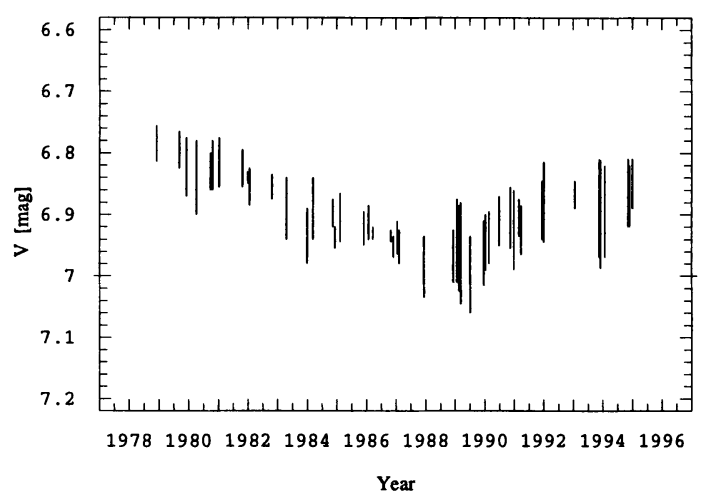

Figure 1. Long-term brightness variation of $\mathrm{AB}$ Dor in the V-band showing a 10-year decline followed by a 6-year rise which began in early 1989 . Each vertical bar represents the range of short-term brightness variation due to spot induced rotational modulation.

jects are known to exhibit long-term changes of their visual brightness by a few tenths of a magnitude (e.g. Cutispoto 1995), little is known about the behaviour of their X-ray luminosity on time scales of several years. I report on an extensive long-term study performed with ROSAT in Sect. 2.

Due to its proximity the Sun is the only star whose distribution of active regions can be observed directly. In the stellar case, indirect techniques such as Doppler imaging (e.g. Vogt et al. 1983; Kürster 1993) or eclipse mapping (Schmitt \& Kürster 1993) must be employed. Unfortunately, with the low spectral resolution of current $\mathrm{X}$-ray observatories it is not yet possible to obtain Doppler images of stellar coronae. Eclipse mapping techniques are applicable only to a small number of suitable objects such as $\alpha \mathrm{CrB}$ (Schmitt \& Kürster 1993). However, with the wealth of data obtained with current X-ray satellites (and especially in the ROSAT all-sky survey), plain $\mathrm{X}$-ray light curves of active stars currently abound. While the spatial information content of a rotationally modulated $\mathrm{X}$-ray light curve is relatively low, a comparison with Doppler images of other layers of the stellar atmosphere can be used to search for correlations between X-ray flux and the visibility of stellar active regions, in an attempt to obtain indirect information on coronal structure. I report on correlated ROSAT X-ray observations and photospheric Doppler imaging in Sect. 3.

\section{Long-term photospheric vs. coronal variability in $A B$ Dor}

\subsection{THE VISUAL BRIGHTNESS}

$\mathrm{AB}$ Dor (= HD 36705) is a rapidly rotating pre-main sequence K0V star $\left(P=12.35 \mathrm{~h} ; v \sin i=93 \mathrm{~km} \mathrm{sec}^{-1}\right)$. For 10 years from 1978 a perpetual 


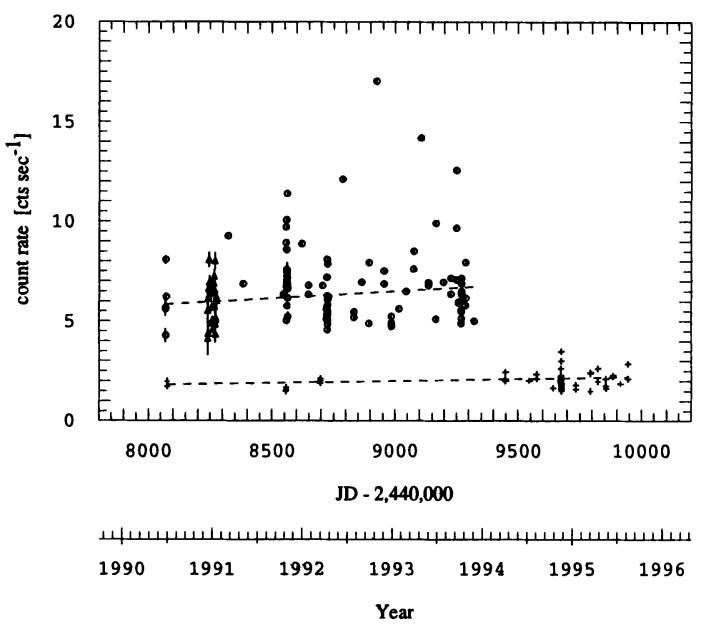

Figure 2. The ROSAT monitoring observations of AB Dor. PSPC measurements are represented by circles (if from pointed observations) or triangles (if from the all-sky survey). Data obtained with the less sensitive HRI are depicted by crosses. Linear least square fits to both the PSPC and HRI data are shown as dashed lines.

decline of its average visual brightness (by $\Delta \mathrm{V} \approx 0 \mathrm{~m} \cdot 2$ ) was observed; in 1989 the reverse trend began. This brightness evolution is shown in Fig. 1 which was adapted from Kürster et al. (1994) and supplemented with recent data (Anders 1994; Bos 1994a, 1994b; Bos et al. 1995). As of now it is unclear whether this variability is cyclic or irregular.

\subsection{THE X-RAY BRIGHTNESS}

In an attempt to find a stellar activity cycle in soft $\mathrm{X}$-rays an ongoing ROSAT monitoring program on AB Dor was begun in mid-1990. AB Dor was selected, because it is a bright $\mathrm{X}$-ray source located near the southern ecliptic pole and therefore visible to ROSAT at all times. It was also chosen because of its pronounced V-band variability and because it resembles a young Sun. In the monitoring program AB Dor is observed once per month for $1-1.5 \mathrm{ksec}$. I complemented the data set by all-sky survey data and by a number of pointed observations of longer duration.

Fig. 2 shows the temporal behaviour of the soft X-ray $(0.1-2.4 \mathrm{kev})$ count rate as observed between mid-1990 and mid-1995. PSPC data are plotted as circles (or triangles if obtained during the all-sky survey); HRI data are depicted by crosses. The systematically lower HRI count rates are a result of the reduced sensitivity of the HRI and do not reflect any stellar property. In 1994 the PSPC became unavailable for this program.

Strong short-term variability is evident (statistical error bars are mostly 


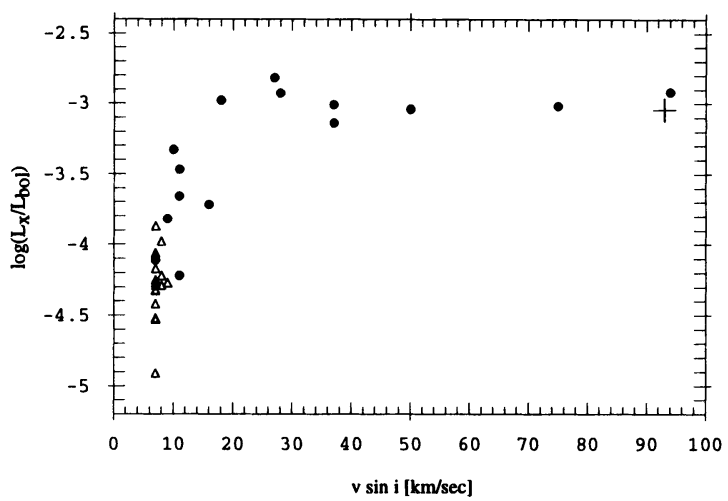

Figure 3. Saturation of coronal X-ray emission in the Pleiades. Plot of $\log \left(L_{\mathbf{x}} / L_{\mathrm{bol}}\right)$ (pertaining to the PSPC band) vs. projected rotational velocity $v \sin i$ for Pleiades members with $0.62<(B-V)_{\circ}<0.85$ (spectral types $\mathrm{G} 2-\mathrm{K} 1$ ). The plot was adapted from Stauffer et al. (1994) to include AB Dor (cross). Genuine measurements are depicted by solid circles whereas triangles represent upper limits to $\log \left(L_{\mathrm{x}} / L_{\mathrm{bol}}\right)$ or $v \sin i$ (or both).

smaller than plot symbols). Formal best fitting straight lines to both the PSPC and the HRI data were plotted together with the data. They have very small slopes, viz. $0.26 \mathrm{cts} \mathrm{sec}^{-1} \mathrm{yr}^{-1}$ (PSPC) and $0.073 \mathrm{cts} \mathrm{sec}-1 \mathrm{yr}^{-1}$ (HRI). Considering the large short-term variability as well as the fact that most measurements are short snapshots which do not cover the whole range of short-term variability present at any given instant, I draw the following conclusion from this data set: A possible long-term trend is marginal, at best, and certainly not comparable to the cyclic soft X-ray variability of the Sun. In the time interval for which ROSAT data are available (after 1990.5) also the $\mathrm{V}$-band flux shows much less systematic change than in previous years. For this reason it is premature to assess any possible correlation between $\mathrm{AB}$ Dor's average $\mathrm{X}$-ray luminosity and its $\mathrm{V}$-band brightness.

The lack of any clear evolution of the X-ray flux supports the concept that the coronae of very active stars are saturated. This concept is employed to explain the observational fact that the $\mathrm{X}$-ray-to-bolometric luminosity ratio $L_{\mathrm{x}} / L_{\mathrm{bol}}$ is constant for the most rapidly rotating late-type stars in young stellar clusters such as the Pleiades, i.e. for samples of stars of equal age. Indications that AB Dor is at the same evolutionary stage as the Pleiades come from the strength of its Lil $\lambda 6707$ absorption line from which an age of $\approx 10^{6}-3 \times 10^{7}$ years was estimated (Ruciński 1982, 1985, Vilhu et al. 1987). This is further supported by AB Dor's membership of the Pleiades moving group (Innis et al. 1986). A comparison of AB Dor's $L_{\mathrm{x}} / L_{\mathrm{bol}}$ with that of the Pleiades G and K-stars is shown in Fig. 3 which was adapted from Stauffer et al. (1994) to include AB Dor (cross). AB Dor finds its place far out in the saturated regime beyond $v \sin i=20 \mathrm{~km} \mathrm{sec}^{-1}$. 

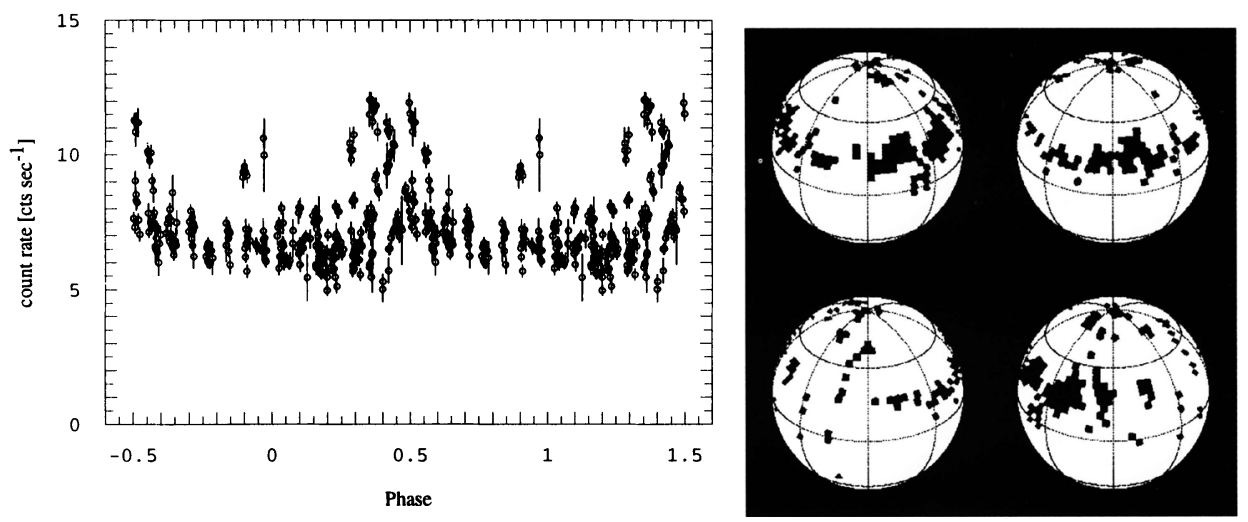

Figure 4. Left: A 46 ksec pointed ROSAT PSPC observation of AB Dor. X-ray light curve folded with rotational period and plotted vs. the phase interval $[-0.5,1.5]$. Note that every data point is plotted twice. Right: Nov 1991 Doppler image (based on CaI 16439) of $\mathrm{AB}$ Dor's photosphere shown at phases $0.0,0.25,0.5,0.75$ (top left to bottom right).

\section{Short-term photospheric vs. coronal variability}

\subsection{AB DOR (= HD 36705)}

Out of the wealth of ROSAT data of AB Dor a $46 \mathrm{ksec}$ pointing carried out within 6 days (Oct 28 -Nov 03,1991 ) is best suited to study short-term variability. Fig. 4a shows the $\mathrm{X}$-ray light curve folded with the rotation period according to the ephemeris $H J D 2,444,296.575+0.51479 \times E$ (Innis et al. 1988). Rotational modulation is present, but with substantial erratic fluctuation superimposed. Assuming the latter to be caused by flares, one can expect to find a well defined, smoothly varying lower envelope to the data points representing the flux from the quiescent corona. This can actually be seen in Fig $4 \mathrm{a}$. The quiescent flux is modulated by $\approx \pm 15 \%$ with the maximum seen at phase $\phi=0.5$. Most of the major flares occur near the same phase indicating that in fact the more active hemisphere is in view.

Fig. 4b shows a contemporaneous Doppler image of AB Dor's photosphere based on high resolution line profiles of $\mathrm{CaI} \lambda 6439$ obtained with the ESO CAT+CES about 3 weeks after the ROSAT observation (Nov 19-28, 1991), i.e. within a time short enough that no major changes of the spot distribution have to be expected (see Kürster et al. 1994; Collier Cameron 1995). For details of the reconstruction technique see Kürster (1993) and Kürster et al. (1994). No pronounced high-latitude spot activity is found. Most of the star spots on AB Dor are grouped into an active belt near latitude $+25^{\circ}$. At $\phi=0.5$, the phase of maximum $\mathrm{X}$-ray flux, there is a gap in the belt implying that the coronal emission is anti-correlated with the visibility of photospheric active regions. 

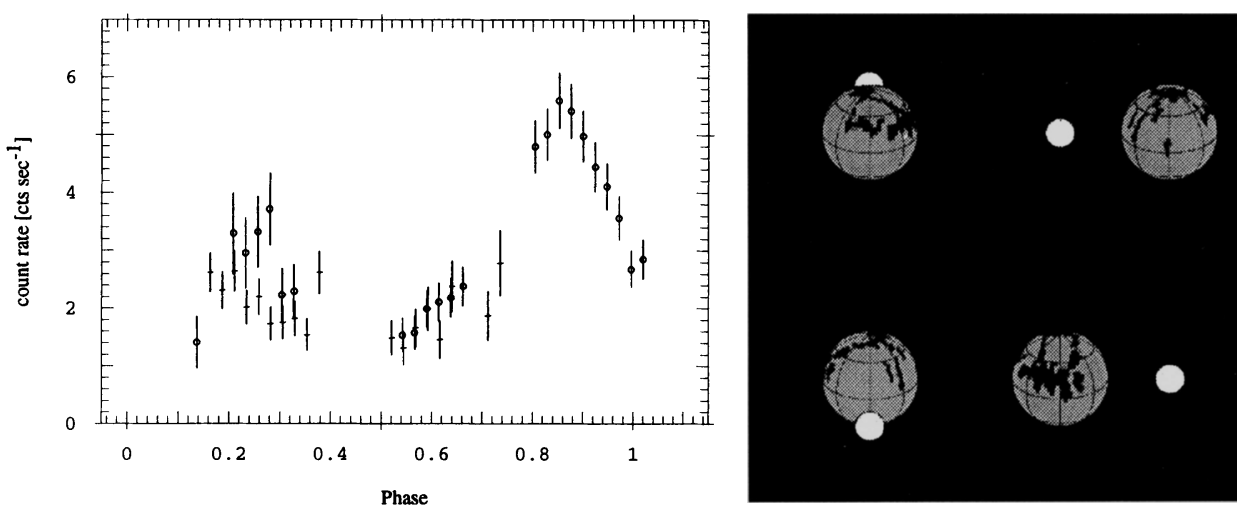

Figure 5. Left: ROSAT all-sky survey PSPC light curve of CF Tuc folded with the orbital/rotational period. Circles: data points from first covered binary cycle; crosses: points from second cycle. Right: Photospheric Doppler image of the CF Tuc K-star (larger component) of Sep 1990 based on CaI $\lambda 6439$. The binary system is shown at phases $0.0,0.25,0.5,0.75$ (top left to bottom right).

\subsection{CF TUC $(=$ HD 5303)}

Fig. 5a shows a ROSAT all-sky survey PSPC light curve of the partially eclipsing RS CVn-type binary CF Tuc (types G0IV+K4IV). It dates from Oct $19-24$, covers $\approx 1.5$ orbital cycles of the synchronized binary, and was folded with the ephemeris $H J D 2,444,555.009+2.797672 \times E$ (period from Budding 1985; phase of conjunction refined with recent optical spectroscopy). A strong modulation is present with minima near the eclipse phases $(\phi=0.0,0.5)$ and a pronounced maximum visible at $\phi=0.8$.

Fig. $5 \mathrm{~b}$ shows the binary as seen at the conjunction and quadrature phases. The photospheric spot pattern on the larger $\mathrm{K}$-star results from a Doppler image (CaI $\lambda 6439$; ESO CAT+CES) obtained 6 weeks before the ROSAT observations (Sep 07-15, 1990). It is dominated by a large feature centered on $\phi=0.8$, i.e. the phase of the $\mathrm{X}$-ray maximum. In addition, there is considerable spot activity near the stellar pole. $\mathrm{V}$-band photometry of CF Tuc of Sep 1990 (Cutispoto, unpublished) reveals a minimum of the out-of-eclipse light curve near $\phi=0.8$, i.e. when the large feature seen in the Doppler image is in view, indicating that the $\mathrm{K}$-star is responsible for the out-of-eclipse variability. However, it is not clear a priori which binary component is the major contributor to the observed $\mathrm{X}$-ray flux. The $\mathrm{X}$-ray light curve suggests a primary eclipse (at $\phi=0.0)$ which would imply an X-ray bright G-star. Kürster \& Dennerl (1993) showed that it is also possible to model the $\mathrm{X}$-ray light curve by pure rotational modulation of two features located in the $\mathrm{K}$-star corona. If the $\mathrm{K}$-star is actually the dominant $\mathrm{X}$-ray source, the striking coincidence of the phases of $\mathrm{X}$ - 

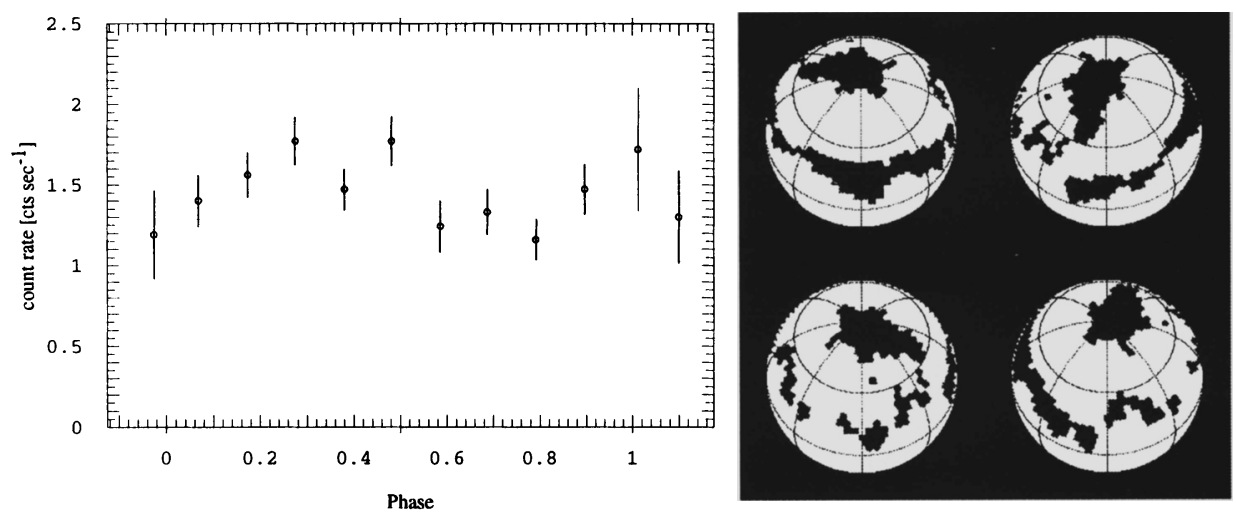

Figure 6. Left: ROSAT all-sky survey PSPC light curve of YY Men plotted vs. time in units of the stellar rotation period. Right: Sep 1990 Doppler image (based on CaI 26439) of YY Men's photosphere shown at phases $0.0,0.25,0.5,0.75$ (top left to bottom right).

ray maximum and visibility of the major spot feature indicates a spatial correlation of the corresponding photospheric and coronal active regions.

\subsection{YY MEN (= HD 32918)}

A third correlated X-ray and photospheric Doppler imaging study was aimed at the single FK Com-type giant YY Men. Fig. 6a shows the ROSAT all-sky survey light curve of Sep 30-Oct 12, 1990. The PSPC count rate is plotted vs. time in units of the stellar rotation phase using the ephemeris $H J D 2,444,155.64+9.5476 \times E$ (Collier 1982). A modulation is seen with a maximum around $\phi=0.3-0.5$ and a minimum near $\phi=0.8$.

A photospheric Doppler image (CaI $\lambda 6439$; ESO CAT+CES) obtained $\approx 3$ weeks before the ROSAT data (Sep 09-19, 1990) is shown in Fig. 6 b. The dominant feature is a large polar spot with an appendage extending towards lower latitudes. A pronounced low-latitude active belt is also seen consisting of many small features. Near $\phi=0.0$ the belt seems to form a continuous band that is probably an artefact of incomplete phase coverage. V-band photometry of Oct 1990 (Cutispoto, unpublished) shows a light curve minimum at $\phi=0.3-0.4$ and a maximum near $\phi=0.8-0.9$. This supports the view that the low-latitude belt actually consists of a rather homogeneous spot distribution not leading to significant brightness change and that the $\mathrm{V}$-band minimum is caused by the passage of the appendage across disk center $(\phi=0.3)$, whereas the maximum is due to the movement of the appendage towards the limb. Obviously, the $\mathrm{X}$-ray modulation is in anti-phase with the $\mathrm{V}$-band variation, i.e. spot visibility is correlated with $\mathrm{X}$-ray flux. The $\mathrm{X}$-ray minimum at $\phi=0.8$ can be explained, if an 
emission region is located beneath the appendage to the polar spot and taken by stellar rotation to the invisible hemisphere at this phase.

\subsection{A POSSIBLE COMMON PICTURE}

For CF Tuc and YY Men a correlation of the X-ray flux with star spot visibility is found while for AB Dor the opposite is observed. The photospheric spot pattern on $\mathrm{AB}$ Dor is oriented predominantly parallel to the equator, whereas in CF Tuc and YY Men spot activity is seen both near the pole and at low latitudes. In an attempt to explain the observational facts by a common (though necessarily non-unique) model, I propose the following concept of large $\mathrm{X}$-ray emitting loops connecting the major spot complexes. An extended loop bridging the gap in the active belt on AB Dor would be oriented parallel to the equator and visible best during phases of minimum spot visibility. Loops on CF Tuc and YY Men connecting the low-latitude features with the polar ones would be oriented meridionally and therefore in view when the dominant spot features are seen.

\section{Acknowledgements}

G. Cutispoto made results from his photometric data available prior to publication. The ROSAT project is supported by the German Bundesministerium für Bildung, Wissenschaft, Forschung und Technologie (BMBF/DARA) and the Max-PlanckGesellschaft. This research used observations collected at ESO.

\section{References}

Anders G.J.: 1994, IBVS 3985

Bos M.: 1994a, IBVS 4111

Bos M.: 1994b, Experimental Astronomy 5, 13

Bos M., Budding E., Hudson G., \& Hudson R.: 1995, IBVS 4203

Budding E.: 1985, IBVS 2729

Collier A.C.: 1982, MNRAS 200, 489

Collier Cameron A.: 1995, MNRAS 275, 534

Cutispoto G.: 1995, A\&AS 111, 507

Innis J.L., Thompson K., \& Coates D.W.: 1986, MNRAS, 223, 183

Innis J.L., Thompson K., Coates D.W., \& Lloyd Evans T.: 1988, MNRAS, 235, 1411.

Kürster M.: 1993, A\&A, 274, 851

Kürster M., \& Dennerl K.: 1993, in Physics of Solar and Stellar Coronae, J. Linsky \& S. Serio (eds.), Lecture Notes in Phys. Ser., Kluwer Dordrecht, p. 443

Kürster M., Schmitt J.H.M.M., \& Cutispoto G.: 1994, A\&A, 289, 899

Ruciński S.M.: 1982, IBVS, Nr. 2203

Ruciński S.M.: 1985, MNRAS, 215, 591

Schmitt J.H.M.M., \& Kürster M.: 1993, Science, Vol. 262, p. 215

Stauffer J.R., Caillault J.-P., Gagné M., Prosser C.F., \& Hartmann L.W.: 1994, ApJS, 91,625

Vilhu O., Gustafsson B., \& Edvardsson B.: 1987, ApJ, 320, 850

Vogt S.S., Penrod G.D., and Hatzes A.P.: 1987, ApJ, 321, 496. 